\title{
Downregulation of lentivirus-mediated ILK RNAi on tractional force generation in human retinal Müller cells
}

\author{
Yu-ping ZHENG ${ }^{1}$, Hui LIU ${ }^{1}$, Hao ZENG ${ }^{2}$, Lei XIONG ${ }^{1}$, Zhao-hui FENG ${ }^{1}$, Nai-xue SUN ${ }^{1, *}$ \\ ${ }^{1}$ Department of Ophthalmology, 2nd Affiliated Hospital, School of Medicine, Xi'an Jiaotong University, Xi'an 710004, China; ${ }^{2}$ Depart- \\ ment of Pathology, Saint Vincent Hospital, Worcester, MA 01608, USA
}

\begin{abstract}
Aim: To investigate the effect of lentivirus-mediated integrin-linked kinase (ILK) RNA interference (RNAi) on human retinal Müller cells transdifferentiation into contractile myofibroblasts.

Methods: A lentiviral vector expressing ILK-specific shRNA was constructed and introduced into cultured retinal Müller cells. Silencing of the ILK gene was identified by real time RT-PCR and Western blot. The Müller cell phenotype change was confirmed by immunodetection of $\alpha$-smooth muscle actin ( $\alpha$-SMA) stress fiber formation. The generation of tractional force was assessed using a tissue culture assay with cells incubated in three-dimensional collagen gels; cell migration was determined by the Boyden chamber method, using $10 \%$ FBS as a chemotactic factor.

Results: Significant decreases in ILK mRNA and protein expression were detected in Müller cells carrying lentiviral ILK-shRNA vector. Cells treated with anti-ILK siRNA showed less $\alpha$-SMA stress fiber formation under hypoxic conditions or cell subcultivation. Lentiviral ILK-shRNA vector transfection also significantly reduced cell migration and cell-mediated gel contraction.

Conclusion: Lentivirus-mediated ILK RNAi decreased cell migration and contractile force generation by inhibiting $\alpha$-SMA stress fiber formation in human retinal Müller cells. This tool might be useful to treat ocular fibroproliferative diseases associated with transdifferentiated Müller cells.
\end{abstract}

Keywords: integrin linked kinase; RNA interference; lentivirus; retinal; $\alpha$-SMA

Acta Pharmacologica Sinica (2009) 30: 1625-1633; doi: 10.1038/aps.2009.154; published online 16 Nov 2009

\section{Introduction}

Epiretinal membrane (ERM) contraction is the leading cause of retinal detachment in proliferative vitreoretinopathy (PVR) and proliferative diabetic retinopathy $(\mathrm{PDR})^{[1,2]}$. Retinal Müller cells (RMCs) are the principal glial cells in the retina and have been detected in fibroproliferative tissues associated with PVR and PDR. These cells might be the source of the tractional force of epiretinal membranes, especially in $\mathrm{PDR}^{[3-5]}$. However, the mechanism of retinal Müller cells migration out of the retina and how they retain the capacity to generate tractional force is not fully understood. A valuable approach to elucidate these mechanism is to trace a-smooth muscle actin (a-SMA)-positive stress fiber formation in retinal Müller cells. As the marker of cell motile activity ${ }^{[6]}$, a-SMA stress fibers have been identified in cells making up the epiretinal membranes, in the context of PDR and $\operatorname{PVR}^{[7,8]}$. In normal retinal

* To whom correspondence should be addressed.

E-mail sunnaixue_xjtu@163.com

Received 2009-06-22 Accepted 2009-09-16
Müller cells, no a-SMA expression can be observed. However, as a result of in vitro culturing, or re-localizing in ERM of PVR and PDR, retinal Müller cells acquire myoid marker a-SMA expression, which causes them to resemble myofibroblasts ${ }^{[8-10]}$. One explanation for the change in retinal Müller cell phenotype is the stimulation of a variety of cytokines and growth factors $^{[10,11]}$, which might come from the injured blood-retina barrier or alternatively, the culture medium. Nonetheless, to date, no study has indicated what kind of downstream signaling is involved in this process.

Integrin-linked kinase (ILK) is a serine/threonine kinase and an important regulator of the epithelial-mesenchymal transition $(\mathrm{EMT})^{[12,13]}$. EMT is a phenomenon characterized in epithelial cells by the loss of epithelial markers and acquisition of a mesenchymal phenotype, which is recognized by the expression of a-SMA ${ }^{[14]}$. Although Müller cells belong to the glia family, their acquisition of a-SMA in some pathological conditions is very similar to EMT, so it is worthwhile examining the role of ILK in the Müller cell phenotype change.

In epithelial cells, ILK is strongly stimulated by transforming 
growth factor-beta (TGF- $\beta$ ) and appears to control the EMT process ${ }^{[15,16]}$. The retinal Müller cells are different from epithelial cells in that their response to TGF- $\beta$ is much weaker ${ }^{[17,18]}$. In this study, we focused on the influence of ILK on the human retinal Müller cell (hRMCs) phenotype change stimulated by hypoxic exposure, because hypoxia is a persistent pathological state for advanced PDR patients with contractile ERM ${ }^{[19,20]}$. We constructed a lentiviral vector expressing ILK-specific shRNA and transfected it into cultured Müller cells, which lead to long-term silencing of ILK. The transduced cells were then examined for a-SMA stress fiber formation, cell migration and tractional force generation, to elucidate the role of ILK in Müller cell transdifferentiation into contractile myofibroblasts.

\section{Materials and methods}

\section{Construction of lentiviral vector expressing ILK-specific shRNA}

Four different ILK-specific target sequences (Figure 1A) were chosen according to online siRNA tools provided by Invitrogen (http://www.invitrogen.com/rnai), using the ILK reference sequence (Gene Bank Accession № NM_004517.2). Double-stranded DNA containing the interference sequences were synthesized according to the structure of a pGCSIL-GFP viral vector (Figure 1B, Gikai gene company, Shanghai, China), and then inserted into linearized vector. All the constructs were cloned and sequenced to confirm their structure. The positive clones were identified as lentiviral vectors that expressed human ILK short hairpin RNA (shRNA), thereafter desig-

A

\begin{tabular}{|l|l|l|}
\hline Marker & Species & TargetSeq \\
\hline ILK-A & Human & CGAAGCTCAACGAGAATCA \\
\hline ILK-B & Human & CTACATGAAGGCACCAATT \\
\hline ILK-C & Human & GCCGTAGTGTAATGATTGA \\
\hline ILK-D & Human & AGAAGCCTGAAGACACAAA \\
\hline
\end{tabular}

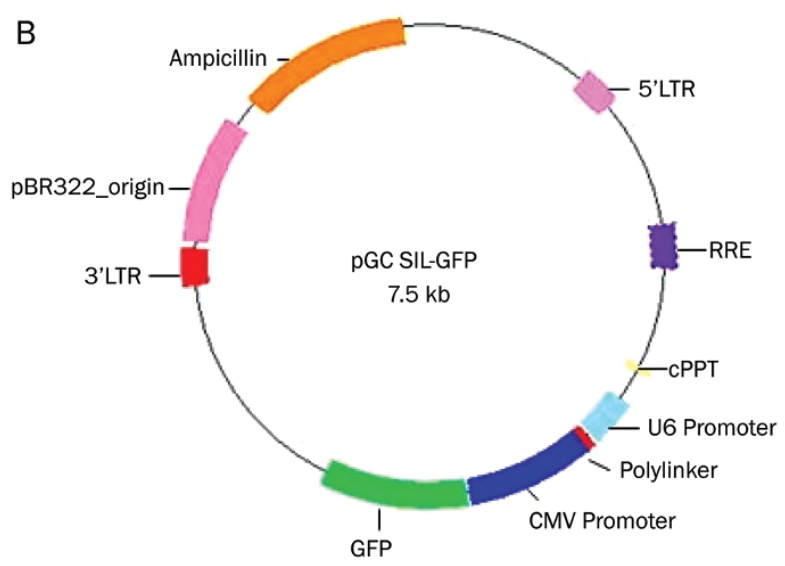

Figure 1. Design of lentiviral siRNAs. (A) Core target recognition sequences of siRNAs from human ILK cDNA. (B) Schematic gene map of the pGCSIL-GFP viral vector into which the siRNA sequences derived from (A) were cloned. nated pGCSIL/ILK-A, pGCSIL/ILK-B, pGCSIL/ILK-C, and pGCSIL/ILK-D, respectively. The four lentiviral vectors were transfected into HEK 293 cells separately to evaluate their RNA interference effects and the pGCSIL/ILK-A (sequence: 5'-CGAAGCTCAACGAGAATCA-3') induced the highest levels of downregulation. So, pGCSIL/ILK-A vector and Viral packaging system (Gikai gene company, Shanghai, China, containing an optimized mixture of two packaging plasmids: pHelper 1.0 vector and pHelper 2.0 vector) were cotransfected into 293 cells to replicate competent lentivirus. Viral supernatant was harvested $48 \mathrm{~h}$ after transfection, filtered through a $0.45-\mathrm{mm}$ cellulose acetate filter and frozen at $-70{ }^{\circ} \mathrm{C}$. The lentivirus(LV) containing the human ILK shRNA-expressing cassette (pGCSIL/ILK-A) was used as a positive control for lentivirus production and denoted as ILK-RNAi-LV in the next experiments. The pGCSIL/U6 mock vector was also packaged and used as negative control, denoted as NC-GFP-LV. Viral concentrations were determined by serial dilutions of the concentrated vector stocks in 293 cells in $96-$ well plates. The number of green fluorescent protein (GFP)-positive cells was measured $4 \mathrm{~d}$ post-transduction under microscopy. The titers averaged to $8 \times 10^{9} \mathrm{TU} / \mathrm{mL}$.

\section{Culture and lentiviral transduction of hRMCs}

The protocol for research involving human tissue complied with the guidelines set forth by the Declaration of Helsinki. Human donor eyes with negative ophthalmic histories were prepared according to procedures previously published in detail $^{[21,22]}$. The eyes were dissected and the retinas were isolated within $4 \mathrm{~h}$ of death. Müller cells were dissociated by papain-DNase digestion and successive trituration, and then kept in a humidified environment of $95 \% \mathrm{O}_{2}, 5 \% \mathrm{CO}_{2}$ at $37^{\circ} \mathrm{C}$ and grown in tissue culture medium (a 1:1 ratio of Dulbecco's modified Eagle's medium and Ham's F12 medium) containing $20 \%$ fetal bovine serum (FBS), the medium were changed twice per week. Cells were identified as Müller glia based on morphology and immunostaining for glutamine synthetase (GS, Santa Cruz Biotechnology Inc, CA, USA), which is typically considered to be a marker of Müller cells and is detected in cultured $\mathrm{RMCs}^{[23]}$. To eliminate the effect of culture time and subcultivation on cell phenotype change, primary cultures were subcultured on the seventh day, and only cells at the first passage which had been cultured for less than $7 \mathrm{~d}$ were used for the subsequent experiments.

For lentiviral transduction, the primary hRMCs were passaged into 6-well plates at a density of $1 \times 10^{5}$ cells/well. When cells reached $30 \%$ confluence (typically on the third day after subculturing), the medium was replaced with $1 \mathrm{~mL}$ of fresh medium containing lentivirus at an MOI of 5 and $6 \mu \mathrm{g} / \mathrm{mL}$ polybrene (Gikai gene company, Shanghai, China). The medium was replaced with fresh medium on the following day.

Analysis of ILK knockdown in hRMCs by quantitative real-time RT-PCR and Western blot

Expression of ILK mRNA in the first passage hRMCs with 
or without vector transfection was detected, with actin as a normalizing control. The specific PCR primer sequences of these genes designed by Beacon designer 2 software were as follows: ILK forward 5'-TCCACCTGCTCCTCATCC-3'; ILK reverse 5'-CCTCATCAATCATTACACTACGG-3'; actin forward: 5'-GGCGGCACCACCATGTACCCT-3'; actin reverse: 5'-AGGGGCCGGACTCGTCATACT-3'. Transduced hRMCs were trypsinized and harvested $5 \mathrm{~d}$ after transduction. Total RNA was isolated using Trizol reagent (Invitrogen-Gibco, Grand Island, NY) and cDNA was acquired according to the M-MLV procedures (Promega) with $2 \mu \mathrm{g}$ of total RNA. Twostep real-time reverse-transcriptase PCR (real-time RT PCR) reactions were performed using the TP800 Real-Time PCR System (TAKARA), which included cycle $1(1 \times)$ : $95^{\circ} \mathrm{C}, 15 \mathrm{~s}$; and cycle $2(45 \times)$ : $95^{\circ} \mathrm{C}, 5 \mathrm{~s} ; 60^{\circ} \mathrm{C}, 30 \mathrm{~s}$. Absorbance data were collected at the end of every extension $\left(60^{\circ} \mathrm{C}\right)$ and graphed using GraphPad PRISM 4.0 software. The real-time PCR data were analyzed by $2^{-\Delta \Delta C t}$.

For Western blot analysis, the cells were washed three times with PBS, homogenized in cell lysis buffer $(50 \mathrm{mmol} / \mathrm{L}$ Tris, $\mathrm{pH} 7.8,150 \mathrm{mmol} / \mathrm{L} \mathrm{NaCl}, 1 \%$ nonidet-40) containing 10 $\mu \mathrm{L} / \mathrm{mL}$ protease inhibitor (Sigma), incubated on ice for $30 \mathrm{~min}$, and then centrifuged for $30 \mathrm{~min}$ at $10000 \mathrm{r} / \mathrm{min}$. The aqueous supernatant was collected and quantified using Bradford Reagent (Sigma). Individual samples, each containing $30 \mu \mathrm{g}$ protein, were separated on a pre-cast $12.5 \%$ SDS polyacrylamide gel in a Tris/ $\mathrm{HCl}$ buffer ( $\mathrm{pH} 7.4$ ) and blotted onto a polyvinylidene fluoride (PVDF) membrane (Bio-Rad, Hercules, CA). The membrane was incubated at room temperature for $1 \mathrm{~h}$ in PBST buffer (PBS pH 7.4, 0.05\% Triton-100), containing 1\% $(w / v)$ bovine serum albumin (BSA) to block nonspecific protein binding sites. After blocking, the blots were probed with a rabbit anti-ILK antibody (Cell Signaling Inc, \#3856, 1:200) overnight at $4{ }^{\circ} \mathrm{C}$, followed by 5 washes with PBST. Blots were then incubated with an anti-rabbit IgG, (1:5000) (Santa Cruz, sc-2357) for $1 \mathrm{~h}$ at room temperature. After washing, the immunoreactive bands were detected using a chemiluminescent substrate (NEN, Life Science). Subsequently, the blots were re-probed with a mouse anti-GAPDH control antibody (1:5000, Santa Cruz, sc-32233).

\section{Immunofluorescence staining for hRMCs ILK expression and $\alpha$-SMA stress fiber formation under hypoxia}

Primary and first passage hRMC cultures with or without lentiviral transduction were incubated in 6-well plates containing glass coverslips at $1 \times 10^{5}$ cells per well. To mimic hypoxia, cells were grown in the presence of $200 \mu \mathrm{mol} / \mathrm{L}$ $\mathrm{CoCl}_{2}$ (Sigma $)^{[24,25]}$, for the designated lengths of time. Control cells were not exposed to hypoxia. The identification of ILK expression and a-SMA stress fiber formation was performed using immunohistochemistry techniques. The coverslip cultures were washed three times with PBS, then fixed in $4 \%$ paraformaldehyde at room temperature for $20 \mathrm{~min}$ and dried in air. After blocking non-specific binding with $0.2 \%$ bovine serum albumin in PBS (PBS-BSA) for $20 \mathrm{~min}$, cells were incubated in the primary antibody, diluted 1:200 in 0.2\% PBS-BSA, at room temperature for $4 \mathrm{~h}$. The primary antibodies used were a monoclonal anti-ILK antibody (1:200) and monoclonal anti-a-SMA (Sigma, Saint Louis, USA, 1:200). The coverslips were washed three times in PBS before addition of secondary antibody conjugated with Cy3 (Sigma, Saint Louis, USA). The coverslips were incubated in the dark for $2 \mathrm{~h}$, washed three times in PBS, and mounted onto glass slides using a mounting medium of $10 \%$ glycerol in PBS. Slides were examined immediately using a fluorescence microscope (Olympus BX50F, Olympus Optical Co Ltd, Tokyo, Japan). Control coverslips, treated with either primary or secondary antibody only, were included in each experiment.

\section{Migration assay}

Migration of the cells was assessed by a modified Boyden chamber method using microchemotaxis chambers ${ }^{[26]}$ (Transwell; Corning Costar, Acton, MA, USA) with polycarbonate membrane filter $\left(8.0-\mu \mathrm{m}\right.$ pore size, $0.33 \mathrm{~cm}^{2}$ growth area). In all experiments, both sides of the membrane were precoated with laminin $(5 \mu \mathrm{g} / \mathrm{mL})$ at $37^{\circ} \mathrm{C}$ for $1 \mathrm{~h}$ and air dried. Medium containing 10\% FBS was added to the lower chambers as chemoattractant. The subconfluent RMCs at passage 1, with or without ILK-RNAi-LV treatment, were trypsinized and resuspended in $2 \%$ FBS to a concentration of $8 \times 10^{5}$ cells $/ \mathrm{mL}$. Cells in the hypoxia group were suspended in $2 \%$ FBS with $200 \mu \mathrm{mol} / \mathrm{L} \mathrm{CoCl}_{2}$ at the same concentration; $30 \mu \mathrm{L}$ of cell suspension (final concentration: $2.4 \times 10^{4}$ cells/well) was added to the upper surface of the membrane. After $24 \mathrm{~h}$ of incubation at $37^{\circ} \mathrm{C}$, the upper surface of the membrane filters was scraped with a cotton swab to remove non-migrated cells and then fixed with $4 \%$ paraformaldehyde for $15 \mathrm{~min}$ at room temperature. Migrated cells which passed through the transwell filter pores and attached on the lower surface of the filters were counted in five non-overlapping fields after nuclear staining with hematoxylin. The experiments were carried out in triplicate.

\section{Collagen I contraction assay}

The contraction assay was performed as previously describ$\mathrm{ed}^{[27]}$, with slight modifications. Cells of the first passage with or without lentivirus transfection were suspended in 1.5 $\mathrm{mg} / \mathrm{mL}$ neutralized collagen I (Cohesion Vitrogen 100; Invitrogen, Palo Alto, CA) at a density of $10^{6}$ cells $/ \mathrm{mL}$ and were then transferred into a 24-well plate (Falcon, Franklin Lakes, NJ) that had been preincubated with a solution of PBS and 5 $\mathrm{mg} / \mathrm{mL}$ BSA overnight. The gel was solidified by incubating at $37{ }^{\circ} \mathrm{C}$ for $90 \mathrm{~min}$, and then the well was flooded with culture medium and $5 \mathrm{mg} / \mathrm{mL}$ BSA, supplemented with buffer or $200 \mu \mathrm{mol} / \mathrm{L} \mathrm{CoCl}_{2}$ to mimic hypoxia. The gels were incubated at $37{ }^{\circ} \mathrm{C}$ with $5 \% \mathrm{CO}_{2}$. The initial gel diameter was 15 $\mathrm{mm}$. The extent of contraction was calculated by subtracting the diameter of the well at a given time point from the initial diameter $(15 \mathrm{~mm})$. Each experimental condition was assayed in triplicate, and at least three independent experiments were performed. 


\section{Results}

Identification of cultured human retinal Müller cells

Phase-contrast images of primary cultured retinal Müller cells on the 3rd day after dissociation are shown in Figure 2A. Cells proliferated from isolated single cells or cell clusters and displayed two types of morphology; most of them were polygonal and flat, while a few were elongated. The cells were identified by staining for glutamine synthetase (GS), a specific marker of Müller cells. Müller cells are the only cells in the retina that express the enzyme glutamine synthetase $(G S)^{[28,29]}$, but expression of GS in cultured Müller cells is transient. Expression appears two days after dissociation and decreases when cells achieve confluence ${ }^{[23]}$. Figure 2B shows GS expression in cultured Müller Cells on the fourth day after dissociation. Positive GS staining was observed in the nuclei of small cells that appeared to be proliferating, while expression appeared to decrease in cells that were large and confluent.
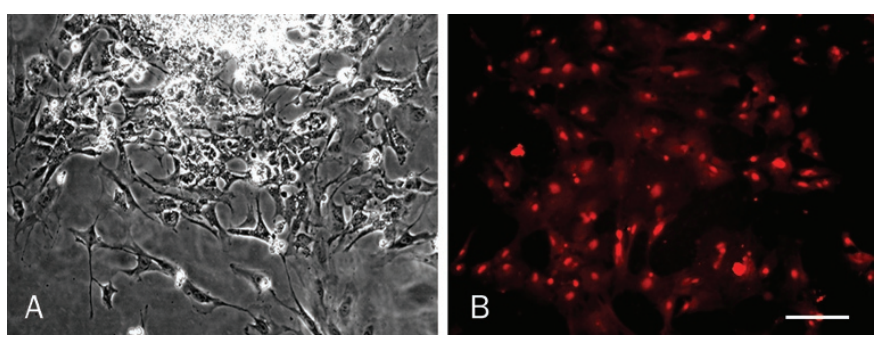

Figure 2. Identification of cultured hRMCs. The primary cultured retinal Müller cells (RMCs) were identified morphologically by phase-contrast images $(A)$ and positive immunofluorescence staining for glutamine synthetase (GS, B). (Scale bar=100 $\mu \mathrm{m})$.

ILK expression of hRMCs was promoted by subcultivation and hypoxic exposure

ILK expression of hRMCs was examined by indirect immunofluorescence. Cells from the first passage of the primary culture were attached to the coverslips and maintained under routine or hypoxic culture conditions for $4 \mathrm{~d}$. These cells were then fixed and stained with ILK-specific monoclonal antibodies. ILK was found to be weakly expressed in the cytoplasm of primary hRMCs under normal conditions (Figure 3A) and intensive perinuclear staining appeared in cells at the first passage (Figure 3B). Exposure of RMCs to hypoxia seemed to alter the expression pattern and level of ILK, with much stronger and extensive fluorescence staining detected in the cytoplasm, both of primary (Figure 3C) and of first-passage cells (Figure 3D).

ILK-RNAi-LV transfection inhibited the expression of ILK mRNA and protein in hRMCs

Among the four candidate target sequences screened, the lentivirus vector containing the human ILK shRNA-expressing cassette (sequence: 5'-CGAAGCTCAACGAGAATCA-3') achieved the greatest efficacy with regard to ILK gene silenc-
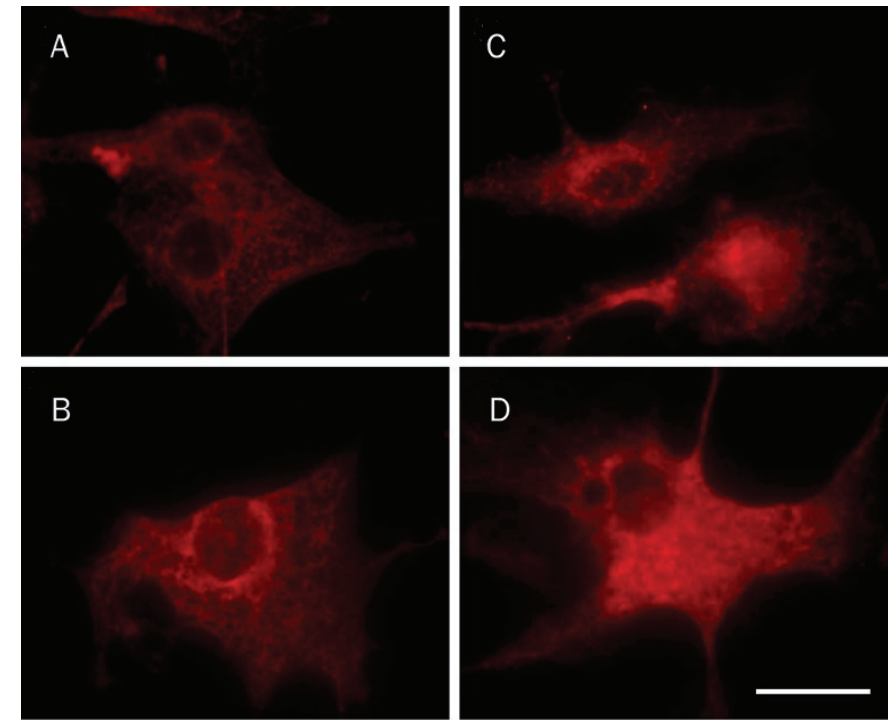

Figure 3. ILK expression in hRMCs. Under normal culture conditions, the primary hRMCs showed weak ILK expression in the cytoplasm (A). The first-passage cells had stronger ILK expression, mainly around the perinuclear area (B). Both primary (C) and first-passage (D) cells exposed to $\mathrm{CoCl}_{2}$ presented much stronger and extensive ILK staining. (Scale bar=50 $\mu \mathrm{m})$.

ing. This construct was therefore denoted as ILK-RNAi-LV; the negative control containing pGCSIL/U6 mock vector only was denoted as NC-GFP-LV. After the ILK-RNAi-LV construct was transfected into hRMCs, ILK mRNA expression levels in transfected cells were compared with those in nontransfected and control-transfected (NC-GFP-LV) hRMCs by quantitative RT-PCR. As a result, cells with ILK-RNAi-LV transfection showed an $82 \%$ reduction in the level of ILK mRNA expression (Table 1 and Figure 4A). To further confirm the specificity of ILK-RNAi-LV-mediated ILK silencing, ILK protein expression was determined by Western blot. As shown in Figure 4B, ILK protein expression in cells transfected with ILK-RNA-LV decreased significantly compared with that of control cells. These results indicate that lentivirus-mediated RNAi was an effective way of modulating ILK expression in cultured hRMCs.

Table 1. Quantitative results of ILK mRNA expression by real-time PCR.

\begin{tabular}{lcc}
\hline & $-\Delta \Delta \mathrm{Ct}$ & $2^{-\Delta \Delta \mathrm{Ct}}$ \\
\hline Control & $-0.20 \pm 0.20$ & $0.99 \pm 0.136$ \\
NC-GFP-LV & $0.00 \pm 0.15$ & $1.00 \pm 0.105$ \\
ILK-RNAi-LV & $3.25 \pm 0.32$ & $0.11 \pm 0.024$ \\
\hline
\end{tabular}

Downregulated expression of ILK following lentivirus-mediated RNAi decreased the $\alpha$-SMA stress fiber formation in hRMCs a-SMA stress fiber formation and localization were observed by indirect immunofluorescence under the microscope. To 


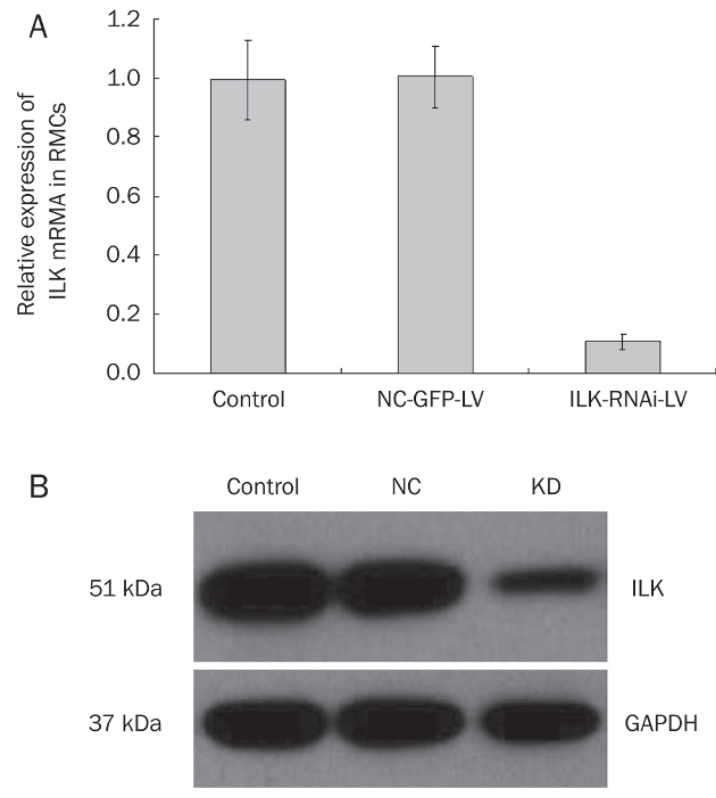

Figure 4. ILK-RNAi-LV transfection silenced the mRNA and protein expression of ILK in hRMCs. (A) Real-time PCR data were analyzed by $2^{-\Delta \triangle C t}$. ILK mRNA expression decreased significantly in cells transfected with ILK-RNAi-LV, as compared to that in untransfected cells (vs control group, $t=13.480$ ) or negative transfection ( vs NC-GFP-LV group, $t=12.163$ ). (B) ILK protein expression detected by Western blot. NC stands for NCGFP-LV group, while KD represented cells with ILK knock down through ILK-RNAi-LV transfection. Data are expressed as means \pm SEM value. $n=3$. ${ }^{\mathrm{c}} P<0.01$.

minimize the phenotype changes in human Muller cells induced by culture time and subcultivation ${ }^{[17]}$, the cells were fixed and stained after attachment to the coverslips and maintained in different culture conditions for less than $7 \mathrm{~d}$. In the primary normal culture, although positive a-SMA staining could be detected at the edge of a few cells, no apparent actin stress fiber formation was observed (Figure 5A). Compared with those in normal conditions, a few primary hRMC cells in the hypoxic condition showed thin actin stress fiber formation in the cytoplasm (Figure 5B). It has been previously reported that hRMC show a-SMA stress fiber formation only when cells are cultured longer than 14 days or subcultured ${ }^{[10,17]}$. In our study, we passaged the primary hRMCs on the seventh day and treated the first-passage cells with lentivirus-mediated ILK RNAi for another four days, with or without hypoxic exposure, before cells were examined again for the presence of a-SMA stress fiber formation. Actin stress fiber formation was detected in some first-passage cells in normal culture conditions (Figure 5C), while much stronger and thicker a-SMA stress fiber formation was observed for those cells cultured under hypoxia (Figure 5D). For the first-passage cells that underwent ILK-RNAi-LV treatment under normal conditions, hRMCs showed less and weaker a-SMA staining compared with those without knock-down transfection (Figure 5E). Hypoxic treatment did not promote their a-SMA stress fiber formation, even though a few thin actin stress fibers could be

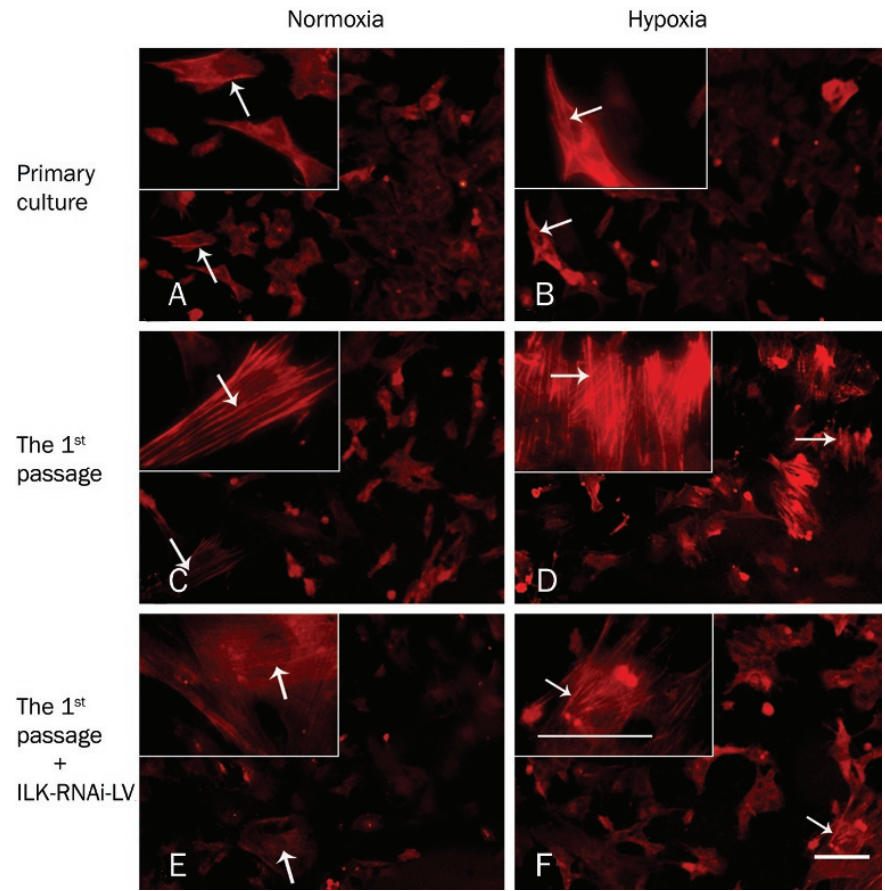

Figure 5. $\alpha$-SMA stress fiber formation in hRMCs. (A) Primary hRMCs under normal culture. $\alpha$-SMA-positive staining is observed only at the edge of a few cells. (B) In primary hRMCs under hypoxic culture, thin $\alpha$-SMA stress fibers appeared in the cytoplasm. (C) In first-passage cells under normal culture, apparent $\alpha$-SMA stress fibers formed in some cells. (D) When the first-passage cells were cultured in hypoxic conditions, stronger and thicker $\alpha$-SMA stress fibers formed in more cells. (E) Weaker $\alpha$-SMA staining in the first-passage cells with ILK-RNAi-LV transfection under normal conditions. (F) The first-passage cells transfected with ILKRNAi-LV did not show more $\alpha$-SMA stress fiber formation when they were exposed to hypoxia. Arrows indicate $\alpha$-SMA-positive stress fibers. (Scale bar $=100 \mu \mathrm{m}$, insets depict expansion of the area indicated by arrows).

detected in the cytoplasm (Figure 5F). Our data indicates that silencing the ILK gene with RNAi might inhibit a-SMA stress fiber formation in hRMCs.

\section{Downregulated expression of ILK by lentivirus-mediated RNAi} decreased hRMC migration

Human retinal Müller cell migration was assayed by the Boyden technique, as described in the Methods section. Cells with motile capacity could migrate through the pores of the transwell filters because of the attraction to 10\% FBS in the lower chamber. In normal culture conditions, hRMCs at the first passage showed a weak ability to migrate (Figure 6A), while hypoxic treatment stimulated cell migration, with three-fold increases in the number of migrating cells (Figure 6B). Cells transfected with ILK-RNAi-LV displayed less migration compared with non-transfected control cells, under both normoxic and hypoxic conditions, as shown in Figure 6C-6D. Cells transfected with NC-GFP-LV, on the other hand, exhibited no change in cell motility, under either normoxic (Figure 6E) or hypoxic (Figure 6F) conditions, compared with non-transfected control hRMC cells. Statistical analyses revealed that 
hypoxic exposure enhanced cell migration $(t=18.741, P<0.001)$. The introduction of ILK-RNAi into cells, however, markedly decreased the cell migration under hypoxia, in comparison to non-transfected cells $(t=25.474, P<0.001)$, or cells transfected with NC-GFP-LV control vector $(t=21.133, P<0.001)$. This dramatic effect on hRMC cell migration associated with ILKRNAi was not observed in those cells maintained under normoxic conditions: all three groups displayed similar but minimal cell migration.

\section{Downregulated expression of ILK by lentivirus-mediated RNAi} decreased the collagen contraction induced by hRMCs

To assess the effect of ILK RNAi on collagen type I gel contraction in first-passage RMCs under hypoxic conditions, cells from passage 1 cultures with or without lentivirus transfection were harvested and suspended in $1.5 \mathrm{mg} / \mathrm{mL}$ neutralized collagen I. The diameter of each gel was measured at the start of the experiment and 24,48 , and $72 \mathrm{~h}$ thereafter. While the changes in gel diameters were not obvious at $24 \mathrm{~h}$ and $48 \mathrm{~h}$ time points, significant gel size shrinkage appeared after $72 \mathrm{~h}$ incubation, especially in wells subjected to hypoxic treatment. Compared with non-transfected and NC-GFP-LV-transfected control groups, cells transfected with ILK RNAi vector showed less of a change in gel size (Figures 7A and 7B). These results indicate that collagen gel contraction induced by hRMCs was augmented by culture time and hypoxic exposure, whereas knockdown of the ILK gene diminished the cell's contractile capacity.

Apparent gel shrinkage and the significant inhibitory effect of ILK RNAi were also observed in the wells under normal conditions, but no significant difference in cell migration was observed. The phenomenon indicated the influence of substrates and culture models on a-SMA stress fiber-related activities. A two-dimension culture with laminin was suitable to examine cell migration, but the contraction assay required a three-dimensional culture model with collagen. The difference between the two assays implied that a collagen type I or three-dimensional culture model might induce more a-SMA stress fiber formation than laminin or two-dimensional culture models. The former yielded more obvious a-SMA stress fiber-

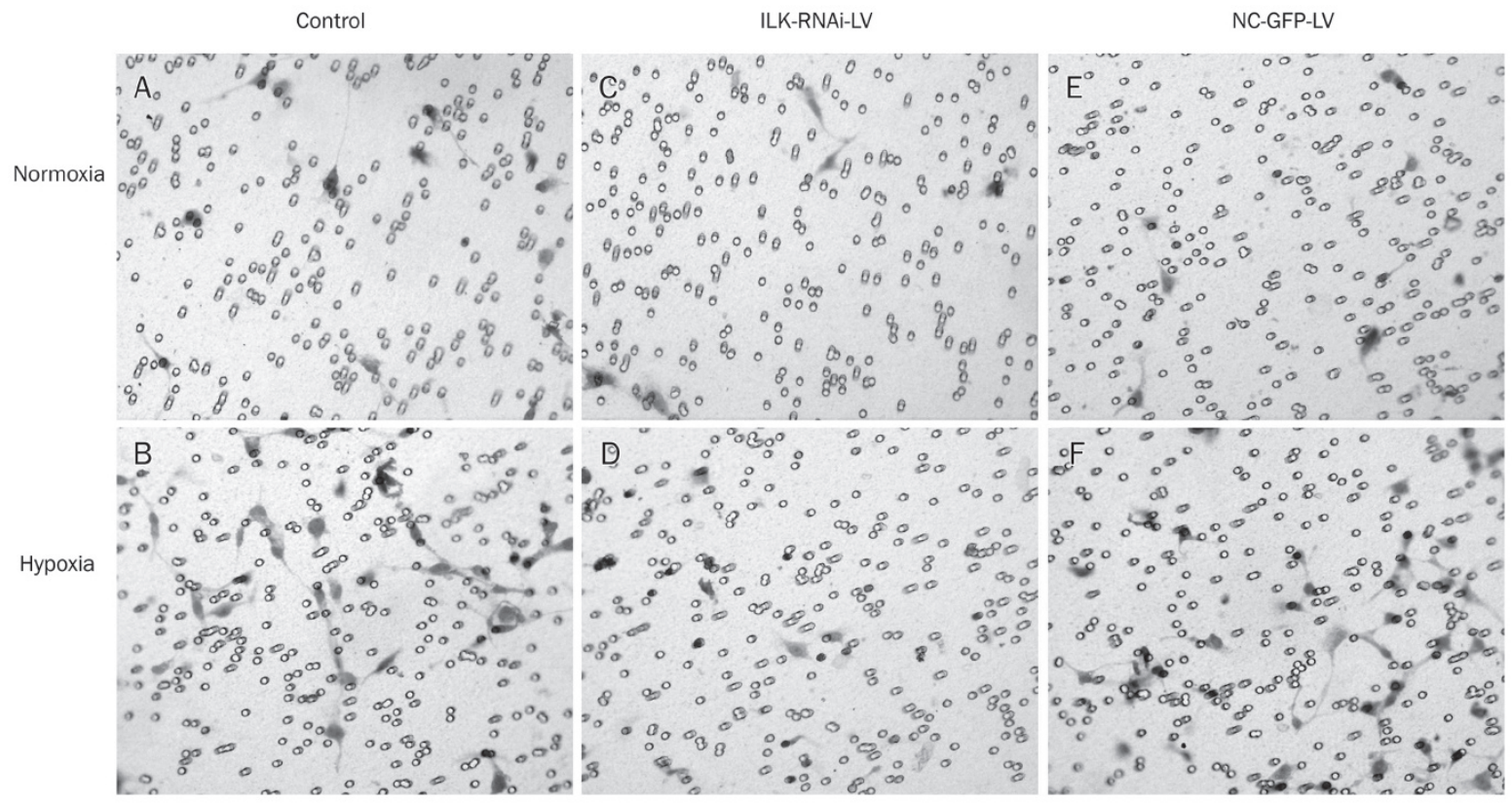

G

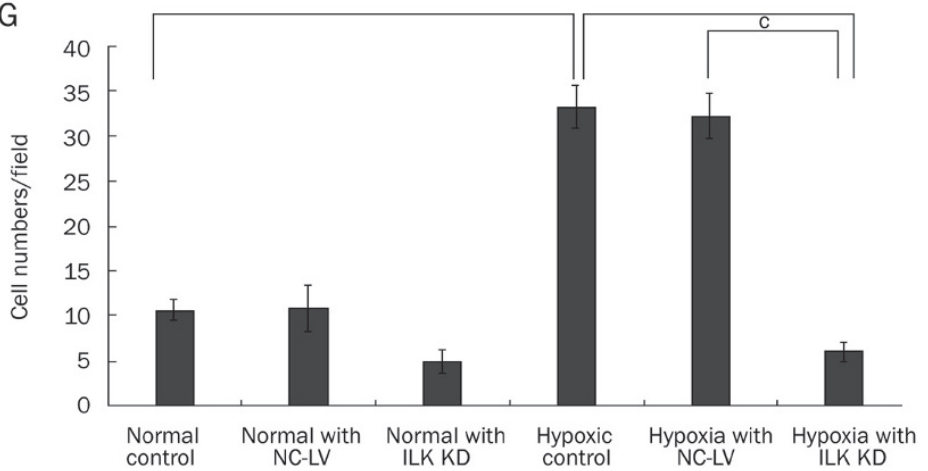

Figure 6. Effect of ILK silencing on the migration of hRMCs. (A) Under normal culture, only a few cells migrate through the pores of Transwell plates. (B) Hypoxic exposure stimulated cell migration; the number of migrating cells increased threefold. Cells transfected with ILK-RNAi-LV showed less migration under normal (C) and hypoxic culture (D) conditions. Cells transfected with NC-GFP-LV exhibited no change in cell motility under normal (E) or hypoxic culture conditions (F), as compared with control groups. (G) Statistical analyses revealed that hypoxic exposure significantly promoted cell migration, and ILK knock down by ILK-RNAi-LV transfection (ILK KD groups) robustly inhibited cell motility. ${ }^{c} P<0.01, n=5$. 


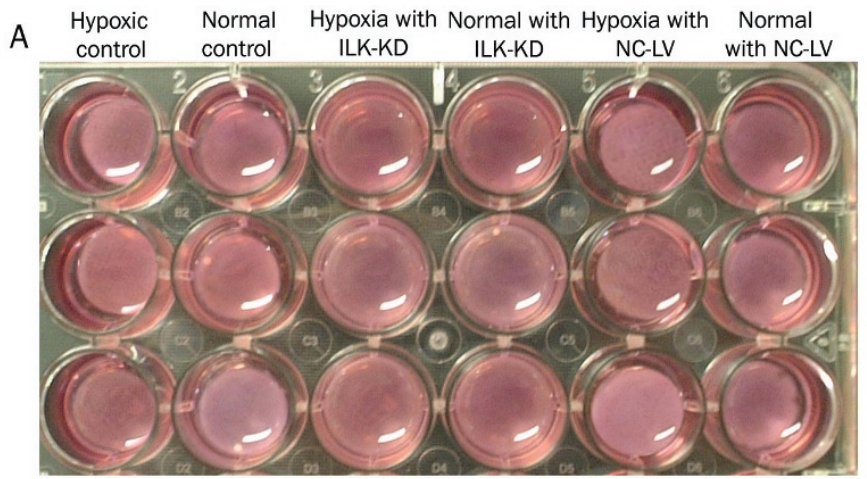

\section{B}

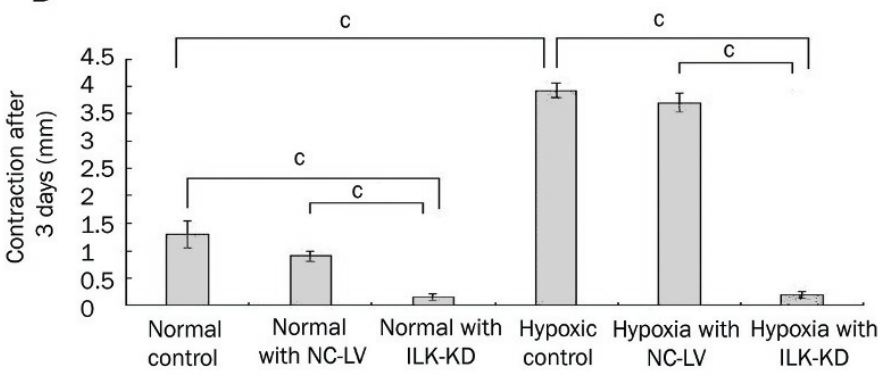

C

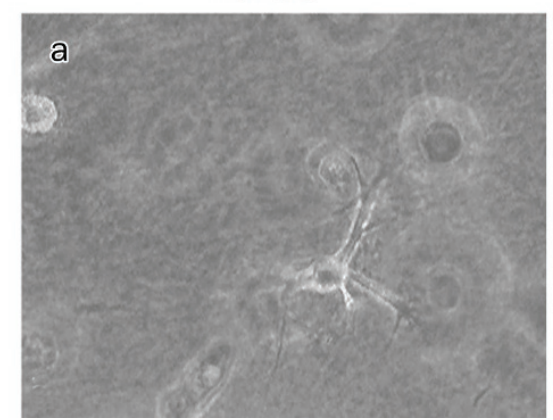

Hypoxia

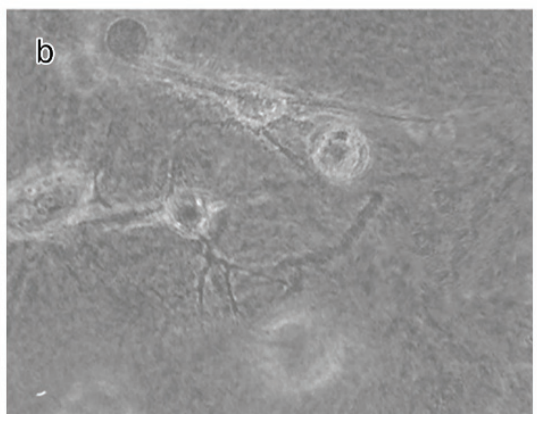

Hypoxia+ILK-RNAi-LV

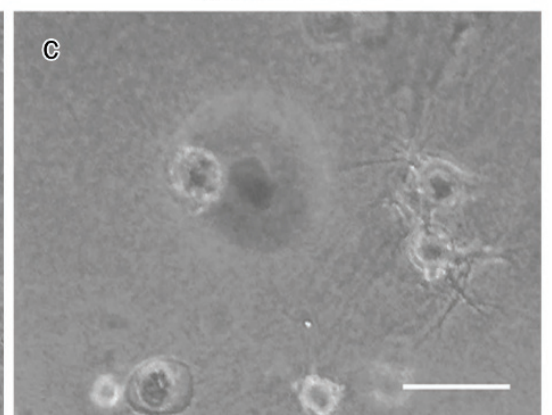

Figure 7. Effect of ILK silencing on the collagen contraction induced by hRMCs. (A) The gels seeded with hRMCs with or without vectors after $72 \mathrm{~h}$ incubation. (B) Statistical analyses showed that hypoxic exposure significantly promoted cell migration ( $t=14.608$, hypoxic control vs normal control), and ILK knock down by ILK-RNAi-LV transfection (ILK KD groups) robustly inhibited hRMC-induced collagen contraction under hypoxic treatment ( $t=59.207$, vs hypoxic control; $t=40.415$, vs negative transfection). Similar inhibition was observed in cells under normal culture. ${ }^{\circ} P<0.01, n=4$. (C) Morphology of hRMCs in collagen gels. (a) Under normal conditions, cells extended short processes in the gel; (b) cells extended longer and stronger processes under hypoxic exposure; (c) cells transfected with ILK RNAi-LV displayed shorter and thinner processes under the same hypoxic treatment. (Scale bar=50 $\mu$ m).

dependent activity, and this effect could also be inhibited by ILK gene knockdown.

The morphology of hRMCs in collagen gels is shown in Figure 7C. Control cells exhibited short processes in gels under normal conditions (Figure 7Ca). Longer and stronger processes extended when cells suffered hypoxic treatment (Figure 7Cb). This hypoxia-induced cell morphology change was abolished, however, by ILK RNAi, with much shorter and thinner process formation, as shown in Figure 7Cc. It is generally believed that cells with long and strong processes are more actively contractile. The cell process changes associated with hypoxia and ILK knockdown were thus in agreement with collagen gel contraction results. The effects of ILK RNAi on collagen gel contraction and cell process changes under hypoxia implied that ILK is involved in RMC tractional force by means of modifying cell morphology and structure.

\section{Discussion}

To date, many published studies have established the key role of contractile myofibroblasts derived from retinal Müller cells or retinal pigment epithelial cells in ocular fibroproliferative disorders, including proliferative vitreoretinopathy (PVR) and proliferative diabetic retinopathy $(\mathrm{PDR})^{[1-5,30]}$. However, the exact mechanism to explain how Müller cells transdif- ferentiate into myofibroblasts remains to be elucidated. The transdifferentiation of Müller cell to myofibroblast is similar to the process of EMT. Both involve a-SMA stress fiber formation, but the stimulators and related signaling involved in the two processes might be different, because the strongest stimulator of EMT, TGF- $\beta$, shows much less of an effect on Müller cell-induced matrix contraction ${ }^{[17,18]}$. In vitro cultured Müller cells undergo phenotype change with prolonged culture time and increasing passage number. It remains to be determined whether this phenomenon is a natural process or rather a result of prolonged stimulation by the small amount of growth factors provided in culture medium. On the other hand, in vivo, phenotypic modification of retinal Müller cells has proven to be the pathological basis for fundus fibroproliferative diseases, which are caused by some abnormality in the vitreous humor or retina.

In this study, we found that hypoxia could stimulate a-SMA stress fiber formation in primary cultured Müller cells, and that this event could be prevented by silencing the ILK gene. Hypoxia is a common and persistent pathological state for patients with advanced $\mathrm{PDR}^{[19,20]}$, which can affect many aspects of Müller cell function, such as proliferation, apoptosis and neuronal support ${ }^{[31-33]}$. Nonetheless, it is difficult to distinguish the effect of hypoxia on Müller cell phenotype in vivo 
because the growth factor imbalance resulting from an injured blood-retina barrier might also exist in the vitreous cavity. Clyde Guidry has proved that IGF-I and PDGF are potent stimuli for Müller cell contraction, and that matrix and integrin $\beta 1$ are involved in the generation of tractional force by Müller cells ${ }^{[17]}$. We examined the expression of integrin- $\beta 1$, PDGF and TGF- $\beta$ receptor in hRMCs under hypoxia, and found that hypoxic exposure could promote integrin- $\beta 1$ expression but had no apparent effect on PDGF or TGF- $\beta$ receptor expression (data not shown). We therefore emphasized the role of ILK in Müller cells phenotype change under hypoxia because ILK interacts with the cytoplasmic tails of the integrin- $\beta 1$ subunits and has been shown to be involved in the regulation of a number of integrin-mediated processes that include cell adhesion, cell shape changes and gene expression ${ }^{[34]}$. In addition to its role in the EMT, the importance of ILK in ocular fibrosis disorders has been identified in RPE cells and in the rabbit PVR model by a recent study ${ }^{[35]}$. However, its role in the transdifferentiation of Müller cells, the other important component of PVR and PDR, has not yet been explored.

In the present study, we provided the first evidence that ILK expression in Müller cells could be promoted by hypoxic exposure and subcultivation, and that increased expression is accompanied by a-SMA stress fiber formation. To further characterize the upregulation of ILK in Müller cells, we downregulated expression of ILK with lentivirus-mediated ILK interference (RNAi) technique. RNAi has recently emerged as a useful genetic tool for silencing gene expression by triggering posttranscriptional degradation of homologous transcripts through a multi-step mechanism involving double-stranded small interfering RNA (siRNA) ${ }^{[36,37]}$. However, direct transfection of siRNAs achieves only a transient reduction in target gene expression in cell culture models ${ }^{[38,39]}$. a-SMA stress fiber formation in cultured cells is a gradual process and affected by many factors including culture duration, medium exchange schedule and cell growth rate. Therefore, studies involving long-term silencing of ILK are necessary. In this study, we used lentivirus to mediate ILK-specific RNA interference. Lentiviral vectors are attractive because they efficiently transduce a wide variety of primary human cells, whether proliferating or quiescent, and can achieve permanent integration into the genomic DNA of transduced cells, thereby enabling long-term modification of cellular phenotype with a single procedure $^{[40,41]}$. We introduced lentiviral vectors expressing ILK-specific shRNA to cultured Müller cells and detected effective silencing of the ILK gene and remarkable reduction of ILK protein expression in transduced cells. After ILK gene silencing, a-SMA stress fiber formation in Müller cells could not be promoted by hypoxic culture or routine subcultivation. Marked decreases in cell migration and tractional force generation were also observed in cells carrying the lentiviral shRNA vector.

Taken together, our study indicated that hypoxia is a stimulus for Müller cell transdifferentiation into myofibroblasts, with ILK acting as an important modulator. By way of lentiviral ILK-specific shRNA-mediated gene silencing, ILK protein expression could be reduced significantly, which in turn diminished cell migration and tractional force generation in Müller cells under hypoxia by decreasing the formation of a-SMA stress fibers. Therefore, lentivirus-mediated ILK RNAi might be useful to treat fibroproliferative diseases associated with transdifferentiated Müller cells.

\section{Acknowledgements}

This work was funded by the National Natural Science Foundation of China (№ 30772598).

\section{Author contribution}

Nai-xue SUN and Yu-ping ZHENG designed research; Yuping ZHENG, Hui LIU, Lei XIONG, and Zhao-hui FENG performed research; Hao ZENG contributed new analytical tools and reagents; Nai-xue SUN and Yu-ping ZHENG analyzed data; Yu-ping ZHENG and Hao ZENG wrote the paper.

\section{References}

1 Pastor JC. Proliferative vitreoretinopathy: an overview. Surv Ophthalmol 1998; 43: 3-18.

2 Sakimoto S, Saito Y, Nakata K, Sakamoto Y, Tatebayashi M. Surgical outcomes of epiretinal membrane removal after successful pars plana vitrectomy for retinal diseases. Jpn J Ophthalmol 2008; 52: 227-30.

3 Nork TM, Wallow IHL, Sramek SJ, Anderson G. Müller cell involvement in proliferative diabetic retinopathy. Arch Ophthalmol 1987; 105: 1424-9.

4 Sramek SJ, Wallow IH, Stevens TS, Nork TM. Immunostaining of preretinal membranes for actin, fibronectin, and glial fibrillary acidic protein. Ophthalmology 1989; 96: 835-41.

5 Guerin CJ, Wolfshagen RW, Eifrig DE, Anderson DH. Immunocytochemical identification of Müller's glia as a component of human epiretinal membranes. Invest Ophthalmol Vis Sci 1990; 31: 1483-91.

6 Paranya G, Vineberg S, Dvorin E, Kaushal S, Roth SJ, Rabkin E, et al. Aortic valve endothelial cells undergo transforming growth factorbeta-mediated and non-transforming growth factor-beta-mediated transdifferentiation in vitro. Am J Pathol 2001; 159: 1335-43.

7 Grisanti S, Guidry C. Transdifferentiation of retinal pigment epithelial cells from epithelial to mesenchymal phenotype. Invest Ophthalmol Vis Sci 1995; 36: 391-405.

8 Dawczynski J, Janz S, Kasper M, Franke S, Königsdörffer E, Augsten $\mathrm{R}$, et al. Histological and immunohistological investigations in human epiretinal membranes. Klin Monatsbl Augenheilkd 2006; 223: 687 90.

9 McGillem GS, Dacheux RF. Rabbit retinal Müller cells undergo antigenic changes in response to experimentally induced proliferative vitreoretinopathy. Exp Eye Res 1999; 68: 617-27.

10 Guidry C. Isolation and characterization of porcine Müller cells: myofibroblastic dedifferentiation in culture. Invest Ophthalmol Vis Sci 1996; 37: 740-52.

11 Hardwick C, Feist R, Morris R, White M, Witherspoon D, Angus R, et al. Tractional force generation by porcine Müller cells: stimulation by growth factors in human vitreous. Invest Ophthalmol Vis Sci 1997; 38: 2053-63.

12 Wu C. ILK interactions. J Cell Sci 2001; 114: 2549-50.

13 Li Y, Yang J, Dai C, Wu C, Liu Y. Role for integrin-linked kinase in mediating tubular epithelial to mesenchymal transition and renal interstitial fibrogenesis. J Clin Invest 2003; 112: 503-16. 
14 Kalluri R, Neilson EG. Epithelial-mesenchymal transition and its implications for fibrosis. J Clin Invest 2003; 112: 1776-84.

15 Lee YI, Kwon YJ, Joo CK. Integrin-linked kinase function is required for transforming growth factor $\beta$-mediated epithelial to mesenchymal transition. Biochem Biophys Res Commun 2004; 316: 997-1001.

16 Burns WC, Kantharidis P, Thomas MC. The role of tubular epithelialmesenchymal transition in progressive kidney disease. Cells Tissues Organs 2007; 185: 222-31.

17 Guidry C, Bradley KM, King JL. Tractional force generation by human müller cells:growth factor responsiveness and integrin receptor involvement. Invest Ophthalmol Vis Sci 2003; 44: 1355-63.

18 Guidry C. Tractional force generation by porcine Müller cells: development and differential stimulation by growth factors. Invest Ophthalmol Vis Sci 1997; 38: 456-68.

19 Abu El-Asrar AM, Missotten L, Geboes K. Expression of hypoxiainducible factor-1alpha and the protein products of its target genes in diabetic fibrovascular epiretinal membranes. Br J Ophthalmol 2007; 91: 822-6.

20 Kaur C, Foulds WS, Ling EA. Blood-retinal barrier in hypoxic ischaemic conditions: basic concepts, clinical features and management. Prog Retin Eye Res 2008; 27: 622-47.

21 Winkler BS, Arnold MJ, Brassell MA, Puro DG. Energy metabolism in human retinal müller cells. Invest Ophthalmol Vis Sci 2000; 41: 3183-90.

22 Puro DG, Yuan JP, Sucher NJ. Activation of NMDA receptor-channels in human retinal Müller cells inhibits inward-rectifying potassium currents. Vis Neurosci 1996; 13: 319-26.

23 McGillem GS, Guidry C, Dacheux RF. Antigenic changes of rabbit retinal Müller cells in culture. Invest Ophthalmol Vis Sci 1998; 39: 1453-61.

24 Kim KS, Rajagopal V, Gonsalves C, Johnson C, Kalra VK. A novel role of hypoxia-inducible factor in cobalt chloride- and hypoxia-mediated expression of IL-8 chemokine in human endothelial cells. J Immunol 2006; 177: 7211-24.

25 Tan XL, Huang XY, Gao WX, Zai Y, Huang QY, Luo YJ, et al. $\mathrm{CoCl}_{2-}$ induced expression of p300 promotes neuronal-like PC12 cell damage. Neurosci Lett 2008; 29: 272-6.

26 Huang W, Wang L, Yuan M, Ma J, Hui Y. Adrenomedullin affects two signal transduction pathways and the migration in retinal pigment epithelial cells. Invest Ophthalmol Vis Sci 2004; 45: 1507-13.

27 Zheng Y, Bando H, Ikuno Y, Oshima Y, Sawa M, Ohji M, et al. Involve- ment of rho-kinase pathway in contractile activity of rabbit RPE cells in vivo and in vitro. Invest Ophthalmol Vis Sci 2004; 45: 668-74.

28 Linser P, Moscona AA. Induction of glutamine synthetase in embryonic neural retina: localization in Müller fibers and dependence on cell interactions. Proc Natl Acad Sci USA 1979; 76: 6476-80.

29 Lewis GP, Erickson PA, Kaska DD, Fisher SK. An immunocytochemical comparison of Müller cells and astrocytes in the cat retina. Exp Eye Res 1988; 47: 839-53.

30 Walshe, R, Esser P, Wiedemann P, Heimann K. Proliferative retinal diseases: myofibroblasts cause chronic vitreoretinal traction. $\mathrm{Br} J$ Ophthalmol 1992; 76: 550-2.

31 Mizutani M, Gerhardinger C, Lorenzi M. Müller cell changes in human diabetic retinopathy. Diabetes 1998; 47: 445-9.

32 Rungger-Brandle E, Dosso AA, Leuenberger PM. Glial reactivity, an early feature of diabetic retinopathy. Invest Ophthalmol Vis Sci 2000; 41: 1971-80.

33 Puro DG. Diabetes-induced dysfunction of retinal Müller cells. Trans Am Ophthalmol Soc 2002; 100: 339-52.

34 Dedhar S, Williams B, Hannigan G. Integrin-linked linase (ILK): a regulator of integrin and growth-factor signaling. Trends Cell Biol 1999; 9: 319-23.

35 Guo L, Yu W, Li X, Zhao G, Liang J, He P, et al. Targeting of integrinlinked kinase with a small interfering RNA suppresses progression of experimental proliferative vitreoretinopathy. Exp Eye Res 2008; 87: 551-60.

36 Hammond SM, Caudy AA, Hannon GJ. Post-transcriptional gene silencing by double-stranded RNA. Nat Rev Genet 2001; 2: 110-9.

37 McManus MT, Petersen CP, Haines BB, Chen J, Sharp PA. Gene silencing using micro-RNA designed hairpins. RNA 2002; 8: 842-50.

38 Daude N, Marella M, Chabry J. Specific inhibition of pathological prion protein accumulation by small interfering RNAs. J Cell Sci 2003; 116: 2775-9.

39 Tilly G, Chapuis J, Vilette D, Laude H, Vilotte JL. Efficient and specific downregulation of prion protein expression by RNAi. Biochem Biophys Res Commun 2003; 305: 548-51.

40 Abbas-Terki T, Blanco-Bose W, Deglon N, Pralong W, Aebischer P. Lentiviral-mediated RNA interference. Hum Gene Ther 2002; 13: 2197-201.

41 Stewart SA, Dykxhoorn DM, Palliser D, Mizuno H, Yu EY, An DS, et al. Lentivirus-delivered stable gene silencing by RNAi in primary cells. RNA 2003; 9: 493-501. 